\title{
N93-22227
}

\section{DEMONSTRATION OF AUTOMATED PROXIMITY AND DOCKING}

TECHNOLOGY

Robert L. Anderson

Roy K. Tsugawa

\section{तré}

Federal Systems Division

1 Space Park Bldg. R11/2337

Redondo Beach, CA 90278

(213)812-2630 Phone

(213)812-8016 FAX
Thomas C. Bryan

NASA

MSFCEB24

Huntsville, AL 35812

(205)544-3550 Phone

(205)544-3801 FAX
$5,-13$

IES ON2Y

$14 / 67 / 2$

9. 2

\section{Statement of technical details of the capability being described}

Automated spacecraft docking operations are being performed using a full scale motion based simulator and an optical sensor. This presentation will discuss the work in progress at TRW and MSFC facilities to study the problem of automated proximity and docking operations. The docking sensor used is the MSFC Optical Sensor and simulation runs are performed using the MSFC Flat Floor Facility. The control algorithms and six degree of freedom (6DOF) simulation software were developed at TRW and integrated into the MSFC facility.

Key issues being studied are the quantification of docking sensor requirements and operational constraints necessary to perform automated docking maneuvers, control algorithms capable of performing automated docking in the presence of sensitive and noisy sensor data, and sensor technologies for automated proximity and docking operations. As part of this study the MSFC sensor characteristics were analyzed and modeled so that off line simulation runs can be performed for control algorithm testing. Our goal is to develop and demonstrate full 6DOF docking capabilities with actual sensors on the MSFC motion based simulator.

We present findings from actual docking simulation runs which show sensor and control loop performance as well as problem areas which require close attention. The evolution of various control algorithms using both phase plane and Clohessy-Wiltshire techniques will be discussed. In addition, 6DOF target acquisition and control strategies will be described.

\section{History of the origins and evolution of the capability}

The initial 6DOF automated control laws were developed and integrated into the motion based simulator at the MSFC Flight Robotics Laboratory in 1989. Since then, added capabilities and new algorithms have continued to be added to the system. The motion based simulation system allows the integration and closed loop demonstration of automated docking system components such as docking sensors, control algorithms, and operational groundrules. Through the use of this facility, we have refined and validated our automated docking system concepts and requirements. 


\section{The level of maturity of the capability}

The motion based simulation facility is fully functional and has been in use each year since its inception. It provides a very powerful testbed for developing and evaluating sensor designs and validating mission design parameters and onboard computer algorithms. As automated docking components are developed, they are tested and validated using the motion based simulation facility.

\section{Test experience and/or experimental results}

The motion based simulations have provided a wealth of data for establishing autodocking and automated proximity operations system requirements. The results of our testing have allowed the characterization of the optical docking sensor performance and has enabled a more robust, higher performance docking sensor design to be created. The experimental results have also contributed to the evolution and design of the control algorithms and operational procedures for automated docking. Based on our findings, we conclude that automated docking is a viable capability which can be implemented using current technology.

\section{Source/sponsorship and current funding estimates}

Over the past several years TRW has continued to invest in the development of automated proximity and docking technologies. We are currently addressing key issues that will result in a mature, automated proximity and docking capability. 